\title{
The role of coacervation and phase transitions in the sandcastle worm adhesive system
}

Russell J. Stewart*, Ching Shuen Wang, and Joshua P. Jones 
1 Abstract. Sandcastle worms, Phragmatopoma Californica (Fewkes), live along the western

2 coast of North America. Individual worms build tubular shells under seawater by gluing

3 together sandgrains and biomineral particles with a multipart adhesive. The glue comprises

4 distinct sets of condensed, oppositely charged polyelectrolytic components-polyphosphates,

5 polysulfates, and polyamines - that are granulated and stored at high concentration in separate

6 cell types. The pre-organized adhesive modules are secreted separately and intact, but rapidly

7 fuse and expand into a crack-penetrating complex fluid. Within $30 \mathrm{~s}$ of secretion into seawater,

8 the fluid adhesive transitions (sets) into a porous solid adhesive joint. The nano- and

9 microstructures of the foamy set adhesive contribute to the strength and toughness of the

10 adhesive joint through several mechanisms. A curing agent (catechol oxidase), co-packaged into

11 both types of adhesive granules, covalently crosslinks the adhesive and becomes a structural

12 component of the final adhesive joint.

18 Keywords: bioadhesive, complex coaceration, condensed polyelectrolytes, sandcastle worm, 19 sabellariidae, Phragmatopoma californica 
1 Introduction. Reef-building sandcastle worms, and related Sabellariidae polycheates, ${ }^{1}$ use underwater glue on a massive scale to geoengineer the world's coastlines. Glued together sabellariid reefs can extend continuously for 100s of kilometers parallel to temperate shorelines worldwide (Fig. 1A).[1,2] The intertidal structures resist wave erosion of the beach, filter and trap sediments, and shelter a rich fauna within the vicinity of the reef.[3,4] Compaction of dead reefs extend the beach foundation seaward and provide a hard base for new colonies.[1] The reefs are glued together one grain of sand at a time. Individual worms meticulously construct tubular shells around their bodies with a secreted bioadhesive and sandgrains or shell fragments captured with ciliated tentacles from the turbulent water column (Fig. 1B,C).[5] In addition to holding the reef together, the glue contains settlement cues that induce planktonic conspecific larva to attach, undergo metamorphosis, and initiate construction of new tubes on existing tubes, thus enlarging the honeycomb-like sandcastle reefs.[6]

The sandcastle worm glue is distinguished from other marine adhesives by the high charge density of its polyelectrolytic components, and by the segregation of positive and negative charges into separate macromolecules. These observations led to the suggestion that the phenomenon of complex coacervation - the spontaneous associative separation of oppositely charged macromolecules into two liquid phases - may play a role in the formation of the adhesive.[7] Complex coacervation has a long history in the field of cell biology. Famously, the spontaneous concentration of primordial organic macromolecules into coacervate protocells was proposed as an intermediate step in the abiotic origin of living cells.[8] In contemporary cell biology, complex coacervation is experiencing a renaissance as a result of the recognition that liquid-liquid phase separation creates transient, phase-defined compartments within the

\footnotetext{
${ }^{1}$ The sandcastle worms described here are Phragmatopoma californica, found off the coast of the western United States. In Europe, closely related species of reef-building sabellariidae polycheates are known as honeycomb worms.
} 
1 cytoplasm.[9] Several examples of abrupt phase transitions of cellular macromolecules, which

2 create switch-like reorganization of the cytoplasm in response to external stimuli, have been

3 recently reported.[10-13]

$4 \quad$ Regulated secretion is another cellular process in which the complex coacervation

5 phenomenon may play a fundamental role. Electrostatic condensation of polyelectrolytic

6 macromolecules into dense fluid granules is a perfect mechanism for packaging and storing large

7 quantities of charged macromolecules, including bioadhesives, that are destined to be secreted in

8 a fluid state in response to a specific signal. Distinct from complex coacervation for sub-cellular

9 compartmentalization, complex coacervation in the regulated secretory system occurs in a

membrane delimited compartment, within which energetic processes control the $\mathrm{pH}$, ion species, ion concentrations, and water content to promote condensation of macromolecules into secretory granules. Secreted biomacromolecules, therefore, are co-adapted both for their extracellular function and for storage within and transit through the regulated secretory pathway, which means they must have suitable physicochemical characteristics for reversible condensation into granules.

The granulated sandcastle glue is a multipart, rapid set, self-initiating adhesive. Its effectiveness as an undersea glue is a product of the cellular sorting and packaging mechanisms, transitions from fluid to solid following secretion, and its final multi-scale porous structure, and not of any one particular amino acid. Here, we describe the entire sandcastle worm adhesive system. Beginning with the adhesive gland structure and physiology, to packaging of the bioadhesive precursors into multiple types of granules, to application of the granulated adhesive to the substrate, to the events of the first few seconds after delivery, to the set and cure mechanisms that lead to the finally structure of the solid foam adhesive. We discuss the role of complex coacervation in adhesive packaging, phase inversion during the setting reactions, 
1 changes in glue morphology during these processes, and the enzyme-catalyzed curing mechanism.
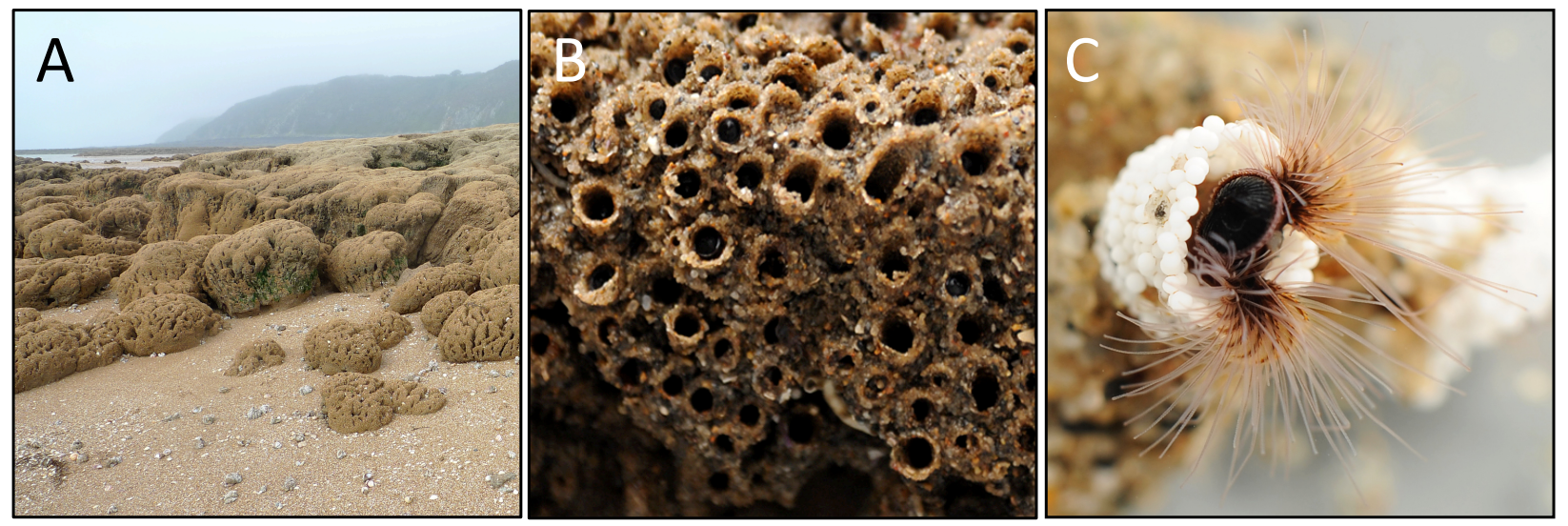

Figure 1. Reef-building sabellariid tubeworms. A) Low tide. Dome-shaped colonies of S. alveolota (foreground) fuse into a tabular surface covering the beach. Photo courtesy of Dr. Jerome Fournier. B) Close up view of a P. californica colony. Each tube was built by an individual worm. C) A worm glued zirconium oxide beads onto the anterior end of its natural tube in a lab aquarium.

Adhesive gland morphology. The gland tissue that produces the sandcastle glue is clustered within the coelomic cavity of the first three parathoracic segments (Fig. 2A,B). As first described by Vovelle,[14] the gland comprises two major cell types that produce either "homogeneous" or "heterogeneous" secretory granules, which are visually distinguished by their morphology and differential staining.[15,16] Heterogeneous granules have a mottled appearance due to the presence of sub-granules (Fig. 3A) and stain darker blue with toluidine blue (TB) than homogeneous granules (Fig. 2C), which appear uniform. Each secretory cell is packed with 100s or 1000s of adhesive granules stored for rapid delivery on demand. The secretory cells are connected to the building organ by long axon-like cellular extensions through which the secretory granules are transported in single file to the surface of the building organ (Fig. 2D,E). The heterogeneous and homogeneous granules, parked just below the surface around the entire circumference of the building organ, remain intact and separated from one another. Each 
2 is no mixing of the separate and distinct adhesive parts within the adhesive gland or building
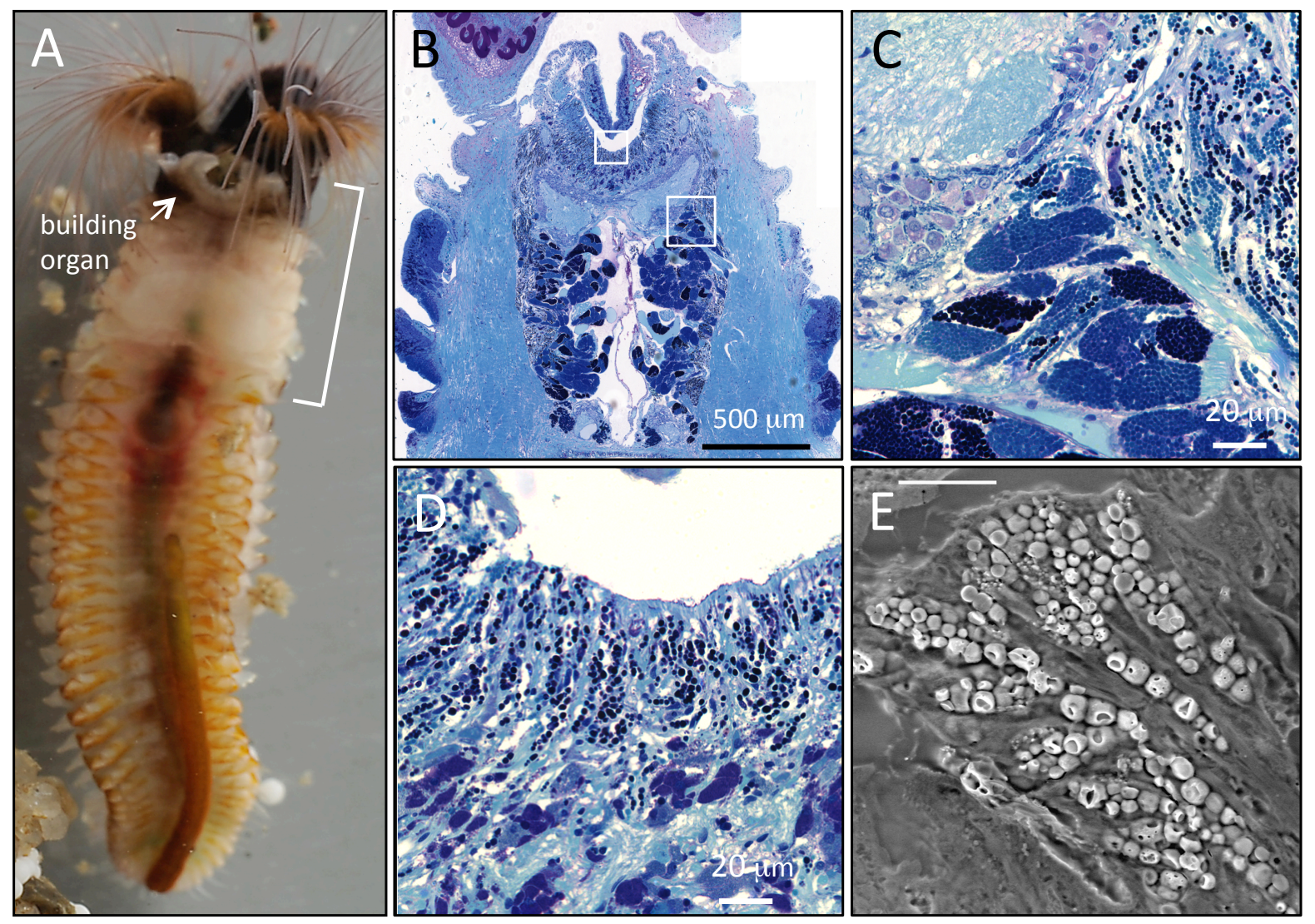

Figure 2. A) $P$. californica out of its tube. B) Coronal section through parathoracic segments indicated by white bracket in A. C) Closer view of boxed region within the adhesive gland. Darker blue cells contain heterogeneous adhesive granules. Lighter blue cells contain homogeneous granules. D) Closer view of boxed region in the building organ. E) SEM of tissu4 just below the surface of the building organ. Reproduced from ref. 23.

Adhesive components and packaging. Sandcastle glue is made of oppositely charged macromolecules distributed in unique pairs between the two types of secretory granules (Table 1).[18] The glue proteins $\mathrm{Pc} 1,2,4,5$ are polycations heavily enriched in lysine $(\mathrm{K})$, histidine $(\mathrm{H})$,

9 glycine $(\mathrm{G})$ and tyrosine residues.[19] The tyrosine residues of Pc1 and Pc2 are posttranslationally hydroxylated to form dihydroxyphenolalanine (DOPA).[20] Pc2 and Pc5 are 

Atlantic coast of Western Europe.[16]

\section{Table 1. Sandcastle glue components}

\begin{tabular}{l|l}
\hline \multicolumn{1}{c|}{ Homogeneous granules } & \multicolumn{1}{|c}{ Heterogeneous granules } \\
\hline Sulfated polysaccharides & Polyphosphate proteins (Pc3B) \\
Polybasic proteins (Pc2, Pc5) & Polyampholytic proteins (Pc3A) \\
Catechol oxidase & Polybasic proteins (Pc1, Pc4) \\
& $\mathrm{Mg}^{2+}$ \\
& Catechol oxidase \\
\hline
\end{tabular}

Blue: polyanions, Purple: polycation and polyanion blocks,

Red: polycations
Red polyanions,

located only in the homogeneous granules along with sulfated polyanions that may counterbalance their charge.[21] Pc1 and 4 are located only in the heterogeneous granules along with polyanionic $\mathrm{Pc} 3 \mathrm{~A}, 3 \mathrm{~B}$, and $\mathrm{Mg}^{2+}$ ions. Both granules contain catechol oxidase that catalyzes crosslinking of DOPA sidechains.[21] Homologs of the P. californica bioadhesive proteins have been identified in Sabellaria alveolata, a tube-building sabellariid found along the

The extremely phosphorylated Pc3 proteins are the most unusual components of the sandcastle worm glue. Pc3B consists entirely of 6-12 residue phosphoserine (pS) blocks separated by single Y residues (Fig. 3A). Pc3A has an N-terminal pS-rich block and a C-terminal block of roughly equal size enriched in $\mathrm{K}$ and arginine (R) residues (Fig. 3A).[7,19,22] Quantitative energy dispersive X-ray spectroscopy of sectioned adhesive glands revealed that phosphorus, and therefore the Pc3A and PC3B proteins, occur exclusively in the subgranules of the heterogeneous granules, co-localized with approximately $0.5 \mathrm{Mg}^{2+}$ ions per phosphate group (Fig. 3 C,D).[23] Only background levels of Ca were detected in the heterogeneous granules and sub-granules. The $\mathrm{pH}$ of the adhesive granules has not been measured and the $\mathrm{pKs}$ of the phosphate groups are unknown making a precise accounting of charge balance impossible. Nevertheless, if the phosphates are monoanionic, the concentration of $\mathrm{Mg}^{2+}$ ions are sufficient to 
1 balance the negative charges on the phosphates. The R-rich cationic block of Pc3A would also

PC-3B:

MKSFTIFAAILVALCYIQISEAG

CCKRYSSSSYSSSSSSSSSSYSSSSSSSSYSSSSSSSS SYSSSSSSSSSSYSSSSSSYSSSSSSSYSSSSSSSSSS YSSSSSSYSSSSSSSSSYSSSSSSSSSYSSSSSSSSSS YSSSSSSYSSSSSSSYSSSSSSSSSSYSSSSSSYSSSS SSSSSYSSSSSSSSSYSSSSSSSYSSSSSSYSSSSSSS SSSSYSSSSSSSSSSYSSSSSSYSSSSSSSSSYSSSSS SSSSYSSSSSSSSSSYSSSSSSSSSSSYSSSSSSYSSS SSSSYSSSSSSSSSSYSSSSSSSYSSSSSSSSSSSYSS SSSSSSSSSSSYSSS

$\mathrm{MW}=30,525$

$\mathrm{pI}=2.5$

B

PC-3A:

MKLLSVFAIVVLAVYITHVEA DSSSSSYSSSSSYSSSSSSSSSSSSSYSSSSSYSSSSSS SYSSSSSYSSSSSYSSSSYSSSSYSSSSYSSSSILTSTS SSDWKRKVPARRVLRTRRFLKCVTRCTLRCILFRSAKT CARKCSRRCLKRVF

$\mathrm{MW}=13,979$

$\mathrm{pI}=2.5$
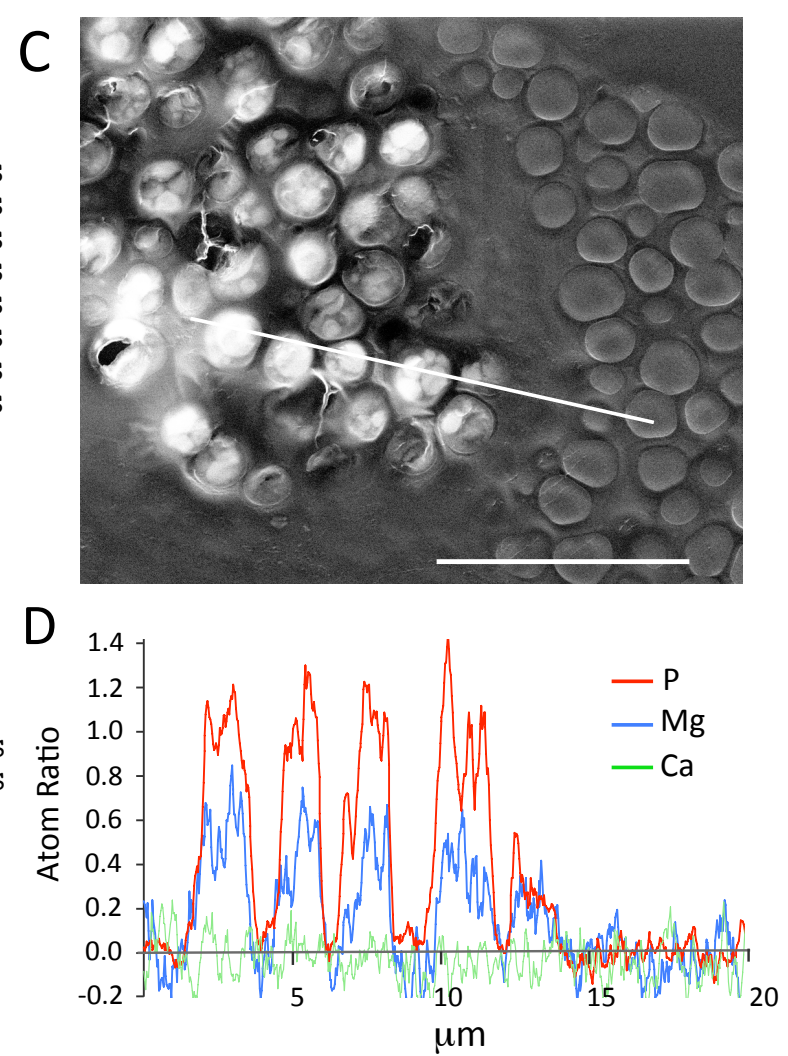

Figure 3. A) Sequence of Pc3B. Secretion signal peptide is red. B) Sequence of Pc3A. with Secretion signal peptide is red. C) SEM image of a tissue section containing homogeneous granules (right) and heterogeneous granules (left). Scale bar $=10 \mu \mathrm{m}$. D) Atom ratios relative to $\mathrm{P}$ in the EDS line scan represented by the white line in $\mathrm{C}$. The scan was from left to right.

5

Synthetic methacrylates copolymers (Fig. 4A) with $~ 70$ mol\% phosphate sidechains

(polyMOEP70) will form clear homogeneous coacervates when mixed with $\mathrm{Mg}^{2+}$ ions at neutral $\mathrm{pH}$ and temperatures less than $20^{\circ} \mathrm{C}$ (Fig. 4B,C).[24] Furthermore, coacervation of polyMOEP and $\mathrm{Mg}^{2+}$ is not affected by salinity; coacervate volume is constant in $\mathrm{NaCl}$ concentrations ranging from 0 to $1.5 \mathrm{M}$ (not shown). Full characterization of the simple coacervation of 
1 polyMOEP with $\mathrm{Mg}^{2+}$ will be presented elsewhere. The demonstration that $\mathrm{Mg}^{2+} / \mathrm{pMOEP}$ forms

2 clear homogeneous fluid coacervates (Fig. 4C) at the temperature and salinity of the ocean

3 provides empirical evidence that it is physicochemically possible that the heterogenous sub-

4 granules of $\mathrm{Mg}^{2+}$ and $\mathrm{Pc} 3 \mathrm{~A} / \mathrm{B}$ are stored as fluid coacervates within the secretory pathway, and

5 are in a fluid state at the time of secretion.
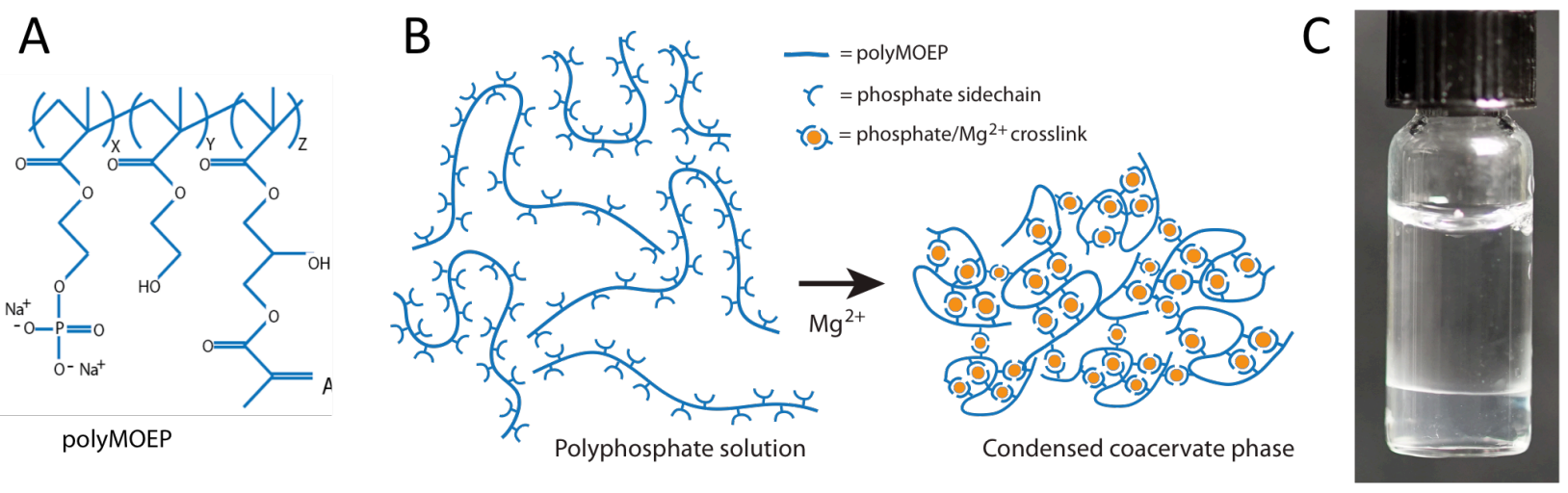

Figure 4. A) Structure of poly(methacryloyloxyethyphosphate (MOEP)-co-hydroxyethyl methacrylate (HEMA)-co-methacryloylglyceryl methacrylate). B) Schematic of $\mathrm{Mg}^{2+}$ induced phase separation (simple coacervation) of pMOEP70. C) The clear homogeneous coacervate of $\mathrm{Mg}^{2+} / \mathrm{pMOEP} 70$ is the dense phase at the bottom of the bottle.

6

Adhesive delivery. The sightless animals depend on the so-called building organ, a dexterous pair of opposed finger-like appendages just below the mouth, for constructing their tubular shells (Fig. 5A). Suspended sandgrains or shell fragments are collected by ciliated tentacles from the water column and transported to the building organ for evaluation. Mineral particles of suitable size ( $\sim 0.5 \mathrm{~mm})$, shape, and surface chemistry are pinched within the building organ 'fingers' and carefully positioned onto the end of the tube and held in place to minimize gaps. Minutes dabs of glue are then applied from the building organ into the narrow gap where particles are in contact. The glue emanates from microscopic pores in the ciliated surface distributed around the entire circumference of the building organ (Fig. 5B,C). The pores frequently occur in close pairs, 

application to the substrate with minimal mixing.[17,23]

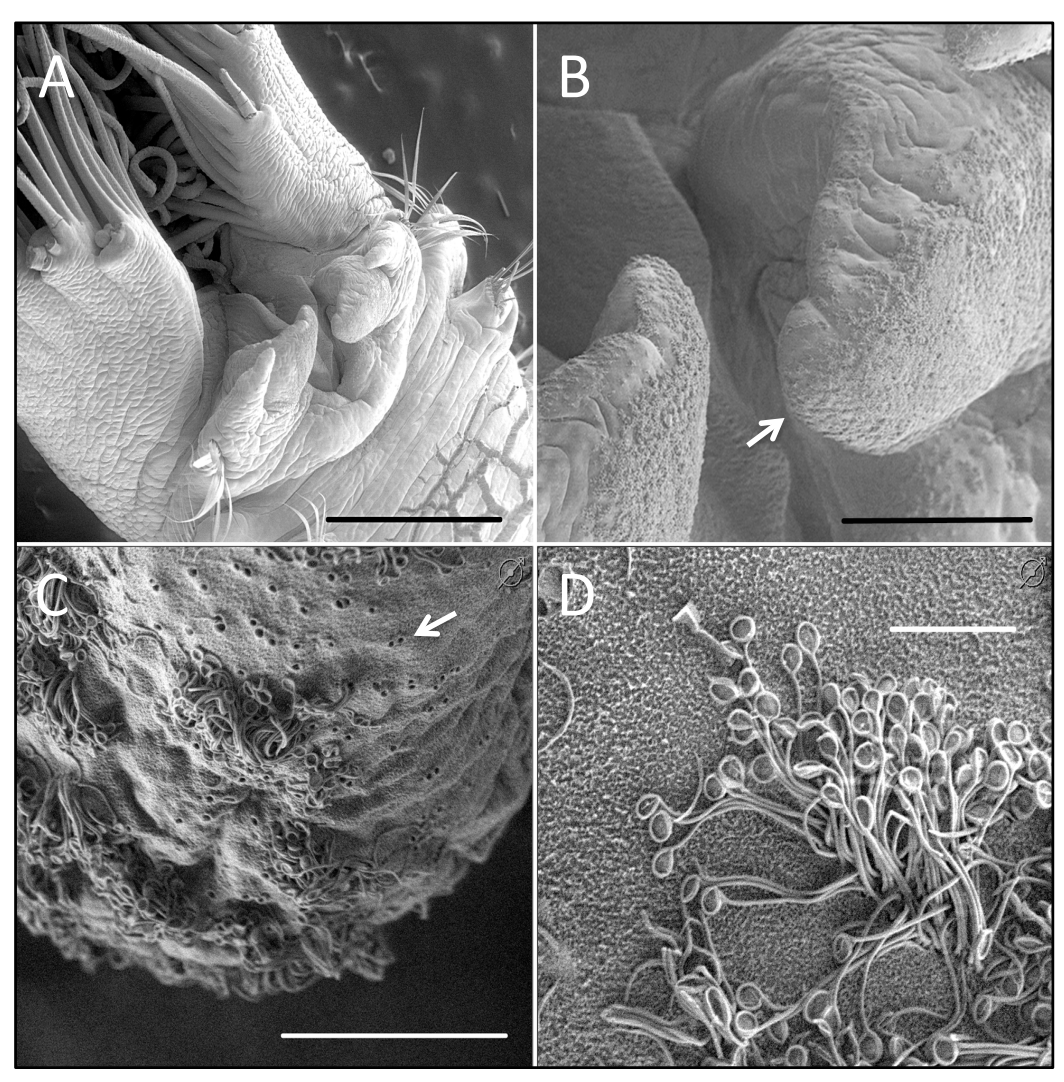

Figure 5. A.) SEM images of the building organ. B.) Closer view of ciliated surface. C.) Region indicated by arrow in B. Adhesive exit pores are indicated by white arrow. D.) Higher magnification of paddle-ended cilia. Scale bars: $1 \mathrm{~mm}, 200,5$, and $20 \mu \mathrm{m}$, respectively. Reproduced from ref. 123.

5

(1)

tempting speculation that one pore is the exit point of a homogeneous cell and the other pore the exit for a heterogeneous cell. Secretion of the two granule types in close proximity to one another would insure uniform distribution within the glue of the separated adhesive parts during

(1)

When worms rebuild their tubes in the laboratory with spherical glass beads, the glue is

applied only where the beads are in contact and at all contact points (Fig. 6A). A supplemental

video (Video S1) reveals how this is accomplished. The worms simultaneously apply two

separate aliquots of glue into the two contact sites between three glass beads. The glue is applied from the side of the contact site and wicks into the narrow gap between the beads forming a 

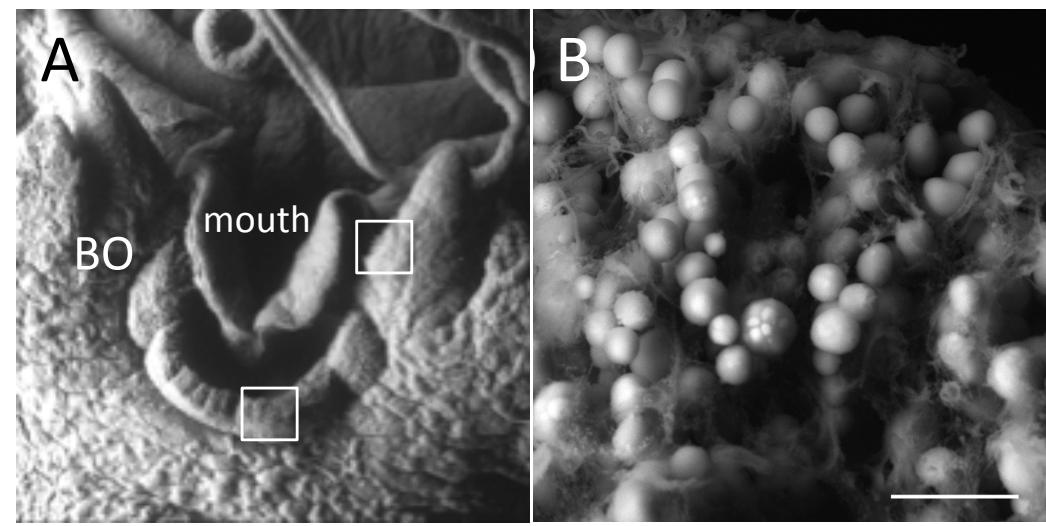
penetrating fluid (Fig. 10C).[23]

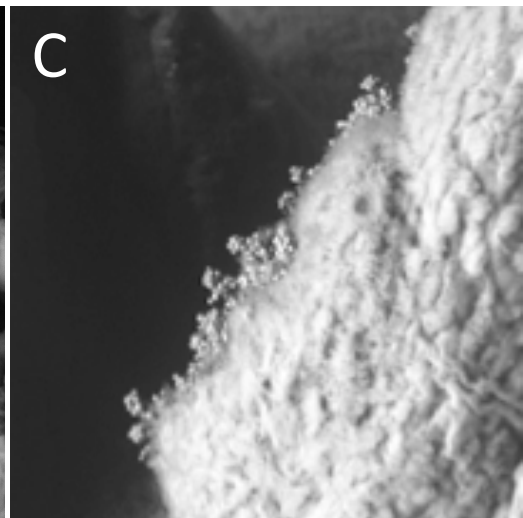

Figure 6. A.) SEM image of the building organ (BO) and mouth. B.) Higher magnification of lower center boxed region. Scale bar $=5 \mu \mathrm{m}$. C.) Higher magnification of upper right boxed region.

13

concave capillary bridge. Strong adhesion of the fluid to the glass surface in seawater is evident from the much less than $90^{\circ}$ contact angle with the bead surface (Fig. XA). The capillary bridge that forms between the particles provides an immediate attractive normal force between the beads. The capillary force has two components: a Laplace pressure difference across the interface between glue and seawater and the interfacial tension that minimizes the interfacial area. $[25,26]$ From the concave surface of the glue joints, the Laplace pressure is lower in the glue phase than in surrounding aqueous phase, which contributes to the normal attractive force. The fluid bridge, however, would contribute little to the compressive or shear moduli of the joint. The adhesive granules were intact when they exited through the surface of the building organ when worms were electrically stimulated to secrete adhesive (Fig. 6).[15,23] Soon thereafter the granules ruptured and the contents fused with little mixing into a lumpy crack

What triggers rupture of the granules after secretion? To investigate, intact granules isolated from dissected adhesive gland tissue in modified Ringer's buffer (430 mM NaCl,10 mM $\mathrm{KCl}, 10 \mathrm{mM} \mathrm{CaCl}_{2}, 50 \mathrm{mM} \mathrm{MgCl} 2,5 \mathrm{mM}$ Tris, $\mathrm{pH}$ 6.5) were surface immobilized in a microscope flow chamber. Solution conditions were then changed to determine if environmental 
1 changes caused granule rupture. In seawater, the granules remained intact and their diameter

2 (volume) was unchanged. Therefore differences in $\mathrm{pH}$, ionic strength, or ionic composition

3 between the interior of the adhesive granules and seawater alone do not trigger granule swelling

4 or rupture (Fig. 7A,B). Likewise, the granules did not swell and rupture in low ionic strength

5 distilled water (Fig. 7C,D). However, in the anionic detergent, sodium dodecyl sulfate (SDS),

6 the granules ruptured and internal vacuoles appeared as the granules rapidly swelled into foamy

7 spheres with roughly two-fold greater diameter than the intact granules (Fig. 7E,F). The swelling

8 was limited as though the swelling forces were balanced by a crosslinked network in the

9 adhesive matrix. Hence, each granule appeared to be a pre-organized, pre-assembled foamy

10 module of the final adhesive that swelled to a fixed volume. This is consistent with the

11 occasional appearance of what appear to be individual granules separated from the main

12 adhesive mass, but which have the porous structure of the bulk adhesive (Fig. 10B, white arrow).

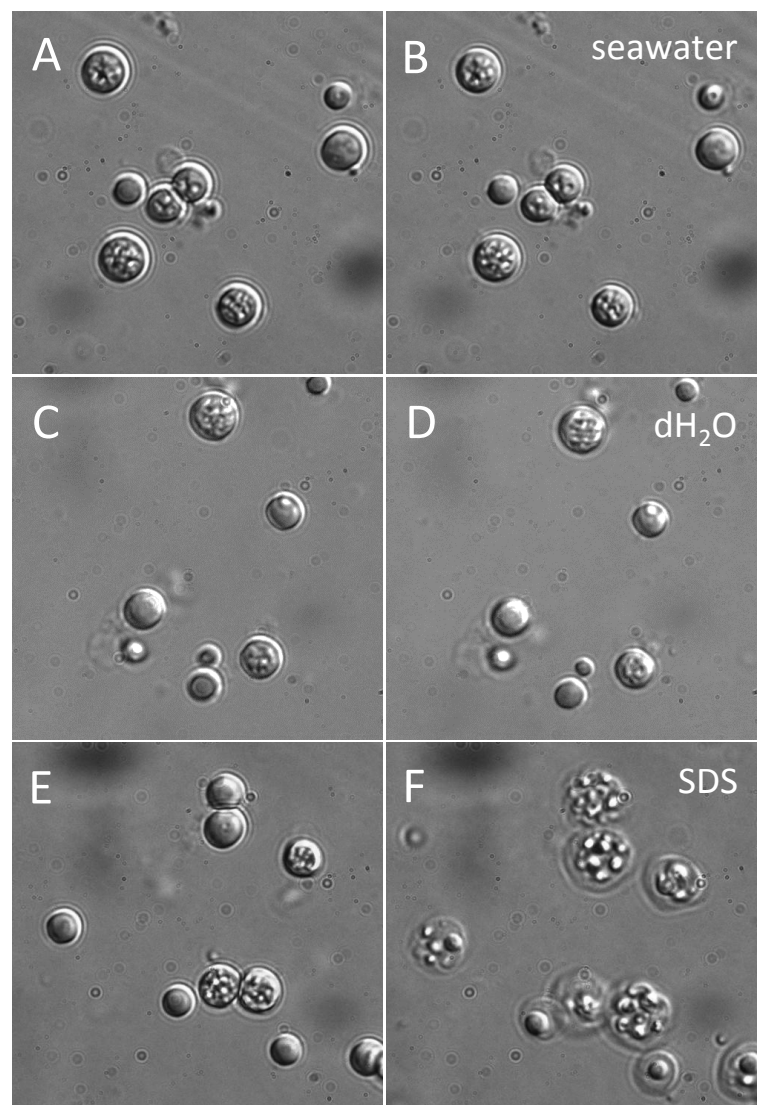

Figure 7. A,C,E.) Isolated adhesive granules immobilized on the surface of a flowchamber in modified Ringer's buffer. B.) Granules in A after $60 \mathrm{~s}$ in artificial seawater, $\mathrm{pH}$ 8.2. D.) Granules in $\mathrm{C}$ after $60 \mathrm{~s}$ in distilled water. F.) Granules in E after $60 \mathrm{~s}$ in $0.05 \% \mathrm{SDS}$. The average diameter of the unruptured granules is 2 $\mu \mathrm{m}$. 

morphology that was distinct from the effect of SDS. The granules swelled until they ruptured,

3 and appeared to lose their contents as they shrunk back to less than the original diameter (Fig

4 8A-C). Within $60 \mathrm{~s}$, the granules appeared to be collapsed, empty shells. The granule response

5 was specific to ATP since GTP had no affect on the granules (Fig. 8D-F). The volume and

6 morphological response to ATP suggests there is an ATPase present in the granules membranes.

7 A likely candidate is a vacuolar $\mathrm{H}^{+}$-ATPase, a group of highly conserved and widely distributed

8 transmembrane enzymes that hydrolyze ATP to pump protons across membranes in eukaryotic

9 cells, including secretory vesicle membranes.[27] The transmembrane $\mathrm{H}^{+}$gradients generated by

$10 \mathrm{H}^{+}$-ATPases, in turn, power transmembrane anti-porters that concentrate components into

11 membrane compartments.[28] The high concentration of $\mathrm{Mg}^{2+}$ ions associated with the $\mathrm{Pc} 3 \mathrm{~A} / \mathrm{B}$

12 coacervates may be transported across the secretory vesicle membranes and concentrated within

13 the secretory granule power $\mathrm{H} \mathrm{H}^{+} / \mathrm{Mg}^{2+}$ anti-porter. When a high concentration of ATP was

14 added to isolated granules (Fig. 8A-C), the $\mathrm{H}^{+}$-ATPase may have over acidified the interior of

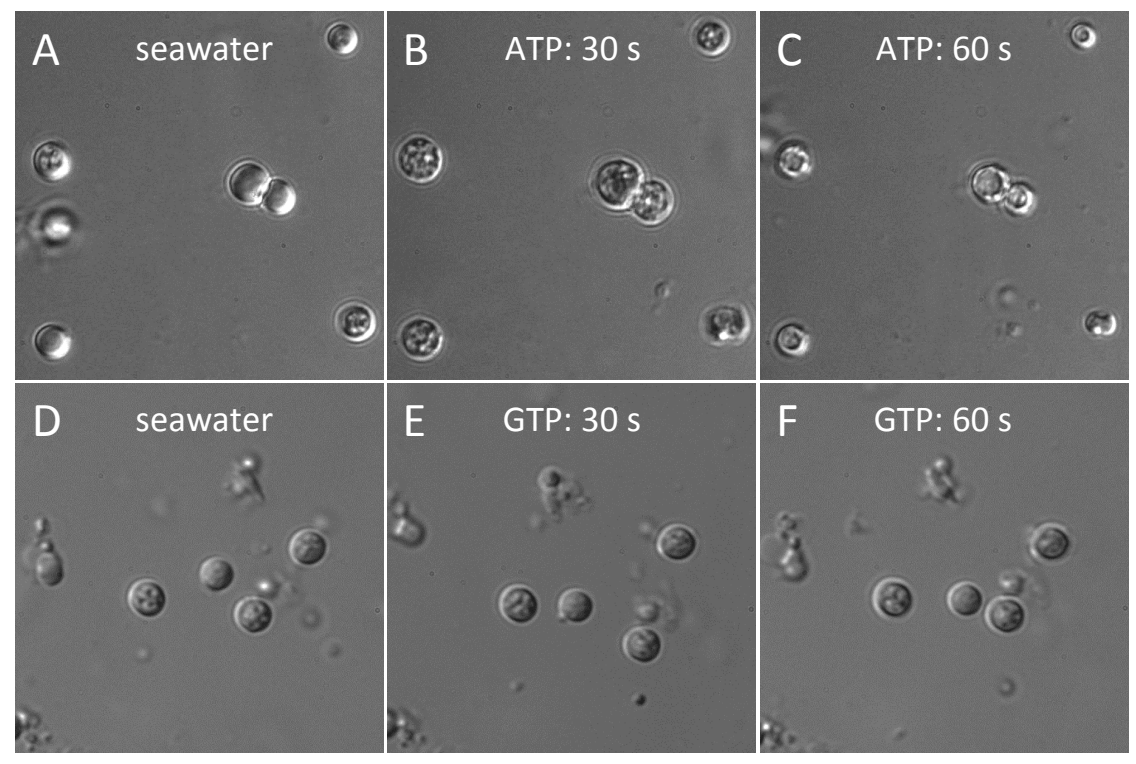

Figure 8. A-C.) Addition of $1 \mathrm{mM}$ ATP to isolated granules in seawater causes granule rupture. D-F.) Addition of $1 \mathrm{mM} \mathrm{GTP}$ to isolated granules in seawater has no effect. 
1 the granules, thus dissolving the coacervated contents, increasing the osmotic pressure within the granules, causing them to burst and release the dissolved contents.

The investigation of bioadhesives in isolated intact granules, as described here, is a promising experimental approach that will allow adhesive proteins and other adhesive components to be studied as packaged in their natural context with their natural counterparts. Although the results presented here are preliminary, they demonstrate that adhesive packaging is an energetic cellular process; a transmembrane ATPase controls the environment inside the adhesive packages, such as $\mathrm{pH}$ and secondarily ion content. This cellular control is likely critical to concentrating the adhesives into granules and controlling their state of matter, such as in the form of fluid coacervates. Understanding the mechanistic details of adhesive packaging and unpackaging is crucial to understanding the sandcastle worm adhesive system, and any natural adhesive system for that matter.

Addition of ATP to isolated granules caused them to rupture, but this is not likely the natural mechanism that ruptures and fuses the granules moments after they are secreted into seawater. Rupture is also not caused by changes in solution conditions, such as $\mathrm{pH}$, on exposure to seawater (Fig. 7A,B). Instead, the granules may be ruptured mechanically by beating of the dense clusters of unique paddle-ended cilia on the surface of the building organ in immediate proximity to the exit pores.[15,23] The worms also apply a back and forth twisting motion while the adhesive is being injected into the gaps of new particles, which may also contribute to mechanically rupturing the granules.[17] Experimentally, when isolated granules were gently sheared between two glass coverslips in seawater, then examined by SEM, the granules were ruptured, had a foamy appearance, and were fused with other granules while largely maintaining their individual integrity (Fig. 9A,B). In other words, there was little mixing of the granule 
1 contents; each granule was a preformed adhesive module fused into the final bulk adhesive.

2 Mechanical rupture of the granules therefore appears to be a plausible mechanism of forming the 3 liquid adhesive immediately after intact granules are secreted.
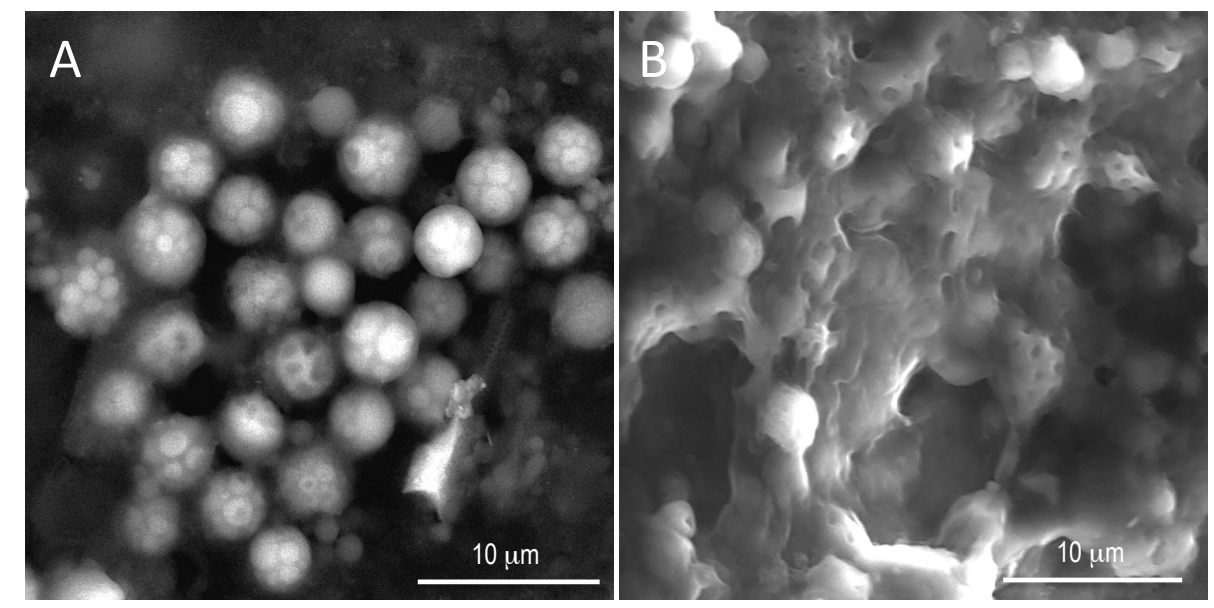

Figure 9. A.) SEM images of isolated granules B.) Granules after shearing between coverslips.

4

Setting mechanisms. Capillary forces can provide an immediate attractive normal force, but to efficiently create a bond that bears shear and compressive loads between mineral particles, the crack penetrating liquid adhesive must quickly solidify and harden after application. In a laboratory aquarium, worms gluing sand grains, glass beads, or bone chips to their tubes slightly wiggled the particles as they were held in place with the building organ as adhesive was applied. The particles were consistently released by the building organ in less than $30 \mathrm{sec}$. From these observations, we inferred that the initial solidification, or set, was sufficient in less than $30 \mathrm{sec}$ for the particles to be self-supporting in the worm's turbulent environment.[17] The transition of the sandcastle glue from a complex fluid to a porous solid-liquid foam with a surface skin and mostly closed cell architecture is triggered by seawater. This is not as simple as the well-known phase inversion processes used to make porous membranes or filaments whereby polymers or polyelectrolytes in a good solvent are cast or printed into a 
1 coagulation bath of poor solvent.[29,30] Looking closely, the closed cells of the glue originate

2 almost entirely from the $\mathrm{Mg}^{2+}$ /polyphosphate sub-granules of the heterogeneous adhesive

3 packets (Fig. 10E,F), an observation also reported by Vovelle.[14] The cells are bounded by

4 chemically distinct, well-defined shells and are embedded in a continuous nanoparticulate,

6 glue using fluorescent anti-pS antibodies revealed that the polyphosphorylated Pc3/B proteins

7 are highly concentrated in the distinct walls of the cells. It appears that during the phase

8 transition to a solid adhesive, the coacervated $\mathrm{Mg}^{2+} /$ polyphosphate in the heterogeneous sub-

9 granules swells and becomes the solid walls of fluid-filled hollow cells.[21] The cells, with an average diameter of $2 \mu \mathrm{m}$, are several fold larger in diameter than the sub-granules.[17] The

11 disposition of the granule phospholipids in the final adhesive is unknown.
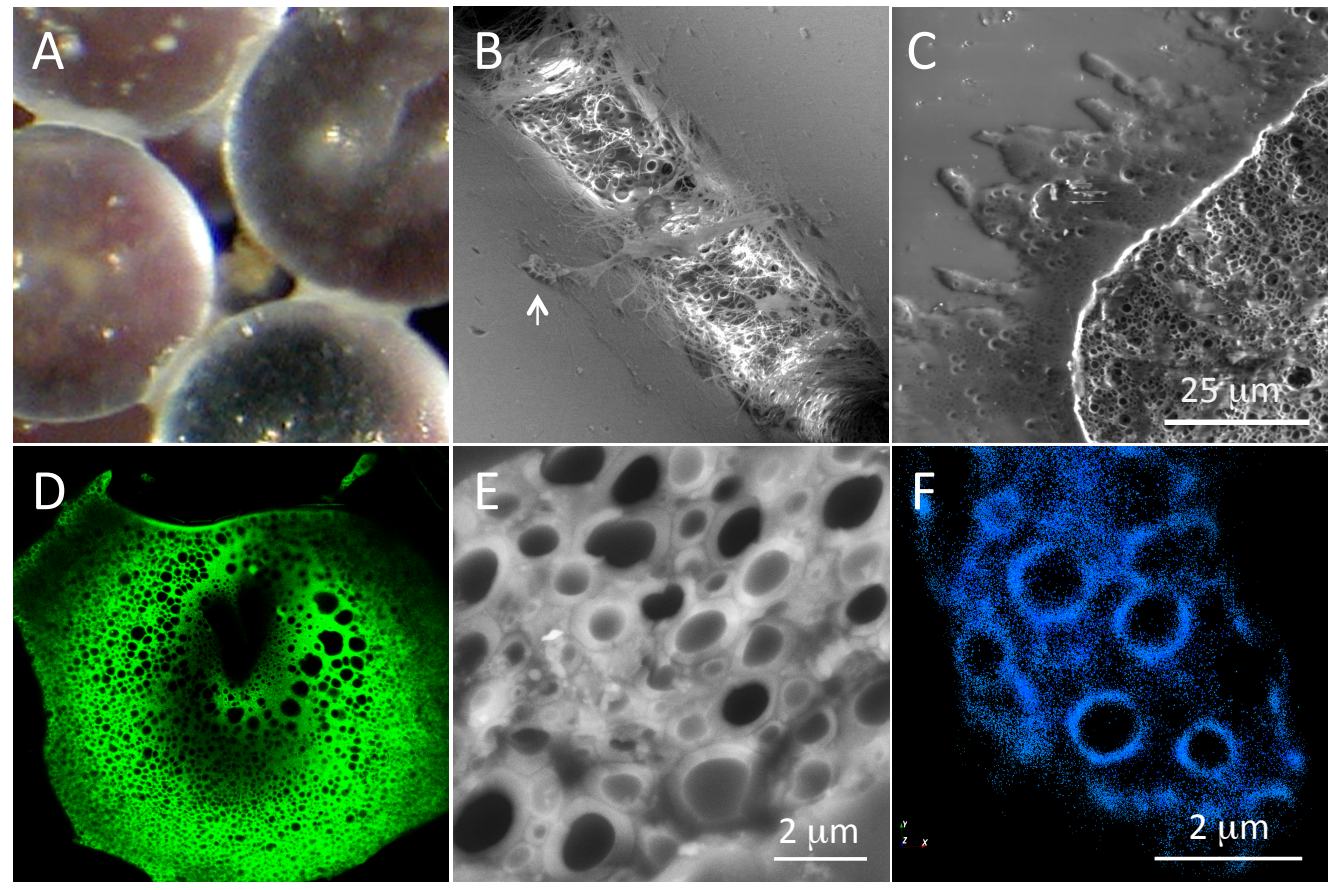

Figure 10. A.) Brightfield image of $0.5 \mathrm{~mm}$ glass beads glued together by a worm in a lab aquarium. B.) SEM image of an adhesive joint between glass beads. C.) SEM image of glue fractured after lyophilization. D.) Optical section of unfixed and hydrated glue using intrinsic fluorescence. E.) SEM image of fixed and sectioned glue. F.) Super-resolution fluorescence micrograph of fixed and sectioned glue using an anti-pS antibody. 
The seawater induced phase transition is driven by a substantial $\mathrm{pH}$ differential between

2 the regulated secretory system $(\mathrm{pH}<6)$ and the ocean $(\mathrm{pH}>8)$ as well as changes in ionic

3 composition. In the sub-granules, the polyphosphates are complexed with $\mathrm{Mg}^{2+}$ only (Fig. 3D),

4 whereas the final adhesive contains both $\mathrm{Mg}^{2+}$ and $\mathrm{Ca}^{2+}$ in a 4:1 molar ratio.[32] Both the

5 increase in $\mathrm{pH}$ and exchange of $\mathrm{Mg}^{2+}$ with $\mathrm{Ca}^{2+}$ would contribute to insolubilization of the

6 polyphosphates.[33] Granule rupture is accompanied by limited swelling, which may be driven

7 by Donnan osmotic pressure that arises from an increase in the internal fixed charge density of

8 the adhesive at the higher $\mathrm{pH}$ of seawater. In particular, the phosphates may transition from

9 mono- to dianionic if the internal and external $\mathrm{pH}$ differential spans $\mathrm{pK}_{\mathrm{a} 2}$. Granule swelling may

10 be limited by a crosslinked network in the matrix around the cells. The osmotic pressure

11 contributes substantially to the compressive and shear moduli of the set glue, and also pre-

12 stresses the walls around the closed cells and the matrix network, which further strengthens the

13 joint.[34] During strain, restricted movement of water through the permeable nanoporous matrix

14 may provide an energy dispersive mechanism that further toughens the joint.[35] In other words,

15 the sandcastle glue is an adhesive structure, not simply an adhesive material.[17] Experiments

16 are in progress to quantify how the composite multiphasic structure and the hydraulic

17 permeability of the sandcastle glue contribute to the mechanical qualities of the adhesive bonds.

18 These structures, toughening mechanisms, and the phase inversion mechanisms that give rise to

19 them can be built into biomimetic underwater adhesives.[36]

20 As an example, we have recently copied the environmentally triggered coacervate phase

21 transitions of the sandcastle glue to create waterborne in situ setting liquid embolic agents.[37]

22 Such agents are widely used in the clinic for minimally invasive control of blood flow.

23 Applications include treatment of arteriovenous malformations, pre-surgical devascularization of 
tumors, and hemorrhage control, to name a few.[38,39] Shear thinning, low viscosity complex coacervates were prepared in hypertonic saline to allow delivery through long, narrow gauge, transarterial catheters. The fluid complex coacervates adhere strongly to blood vessel walls, which allows precise placement of the embolic agent. As they equilibrate toward physiological ionic strength, the embolic coacervates transition into ionic gels as the electrostatic interactions between oppositely charged polyelectrolytes are less shielded by counter ions. The adherent gels occlude the blood vessel and block the flow of blood.[37]

The transition from fluid to solid is accompanied by the formation of a discontinuous porous phase (Fig. 11). As with the fluid-filled multiphasic structure of the natural sandcastle glue (Fig. 10D), the phase inversion mechanism and the resulting multiphasic porous structure of the in situ setting embolic coacervates may contribute osmotic pre-stressing and hydraulic toughening mechanisms to the set adhesive. A more complete description of the ionic strength induced phase inversion of the adhesive coacervates, the final adhesive structure, and mechanics will be presented elsewhere. Inspired by the setting mechanisms and multiphasic structure of the sandcastle glue, coacervate phase inversions in water, without organic solvents, may provide a pathway to the manufacture of novel nano- and micro-structured membranes, coatings, and adhesives for medical applications.

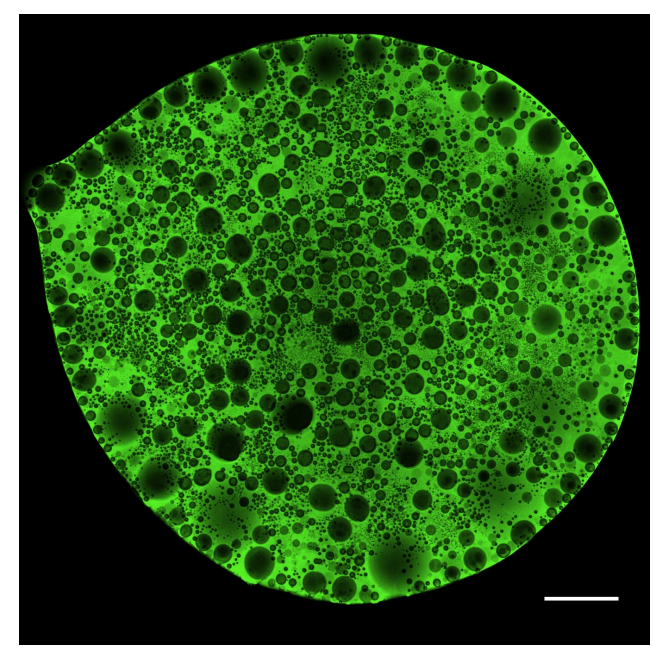

Figure 11. Confocal image of adhesive embolic set in $150 \mathrm{mM} \mathrm{NaCl}$. FITC-labelled protamine sulfate and sodium hexaphosphate at a 1:1 charge ratio form clear fluid complex coacervates in $1200 \mathrm{mM} \mathrm{NaCl}$. Ten microliters of the coacervate was applied to a glass slide submerged in 150 $\mathrm{mM} \mathrm{NaCl}$. Fluorescent images were made with a Nikon A1R confocal microscope using a $4 \mathrm{x}$ objective. Scale bar $=500 \mu \mathrm{m}$. 
1 Curing mechanism. The sandcastle glue turns reddish brown, indicative of quinonic crosslinking, over the course of a few hours after secretion.[17] Covalent crosslinking, or curing, of the adhesive provides additional strength to the joint. Covalent curing is catalyzed by a catechol oxidase enzyme that is co-packaged into both the homogeneous and heterogeneous granules.[21] Catechol oxidases are type 3 copper proteins with binuclear $\mathrm{Cu}$ active sites that catalyze oxidation of diphenols.[40] The $\mathrm{Cu}$ concentration in the sandcastle glue is $0.7 \mathrm{ppm}$, which is several orders of magnitude higher than the $\mathrm{Cu}$ concentration in natural seawater.[41] Therefore, the $\mathrm{Cu}$ is most likely present in the adhesive before secretion in the active site of the catechol oxidase. The high $\mathrm{Cu}$ content suggests that catechol oxidase is a major protein constituent of the adhesive joints. The ratio of $\mathrm{Cu}$ to $\mathrm{P}$ is approximately 1:2000, which corresponds to a ratio of catechol oxidase to $\mathrm{Pc} 3 \mathrm{~A} / \mathrm{B}$ on the order of $1: 10$, estimating that on average Pc3A/B have approximately 100 phosphate groups and catechol oxidase has two copper atoms. The high concentration of enzyme co-packaged within both types of micron-sized packets ensures rapid and homogeneous crosslinking of the modular, multipart adhesive without the need for the enzyme to diffuse through or be mixed into the adhesive. The enzyme remains embedded and active within the glue long after the glue has fully cured.[21] As a major constituent of the glue, the catechol oxidase enzyme is likely an integral structural element of the glue.

We have theorized that complex coacervation may also play a role in the regulation of the catechol oxidase enzyme.[18] The pro-enzyme contains a 74 amino acid pro-peptide, which is unstructured and polyampholytic; 31 of 74 residues are charged at neutral $\mathrm{pH}$. It is possible that the enzyme is electrostatically inactivated before secretion by association of the propeptide with the other polyelectrolytic components in the secretory granules. The highly charged propeptide could also play a role in electrostatically condensing the latent catechol oxidase into the 
1 granulated adhesive. Upon granule lysis in seawater, the enzyme may be activated by exchange

2 and rearrangement of electrostatic associations. There are numerous examples of latent plant

3 catechol oxidases that are activated by anionic detergents that bind specific sites of the

4 enzyme.[42] These precedents provide empirical evidence that electrostatic interactions can

5 reversibly switch enzymes on and off.

6 Summary. Sandcastle worms construct reefs under turbulent seawater by joining irregularly

7 shaped particles into external tubular structures. The nature of the process requires a fluid state

8 adhesive, denser than seawater, that will penetrate the narrow spaces between closely opposed

9 particles while not dissolving into the ocean. The fluid adhesive provides an immediate capillary

10 force between the particles, but must rapidly transition into a solid state to resist shear and

11 compressive forces in the turbulent environment. To accomplish this feat, the sandcastle glue is

12 granulated under energetic cellular control into two types of pre-formed, pre-organized adhesive

13 modules. The granules fuse into a crack penetrating fluid adhesive coacervate. Seawater is a poor

14 solvent for the fluid adhesive, which leads to outside inward hardening of the fluid coacervate

15 through microphase separation and insolubilization. Many questions remain about the phase

16 transitions that occur in the glue during first few seconds after secretion and the contributions of

17 the final composite porous structure to mechanical attributes of the sandcastle glue. Isolation of

18 intact bioadhesive granules, as described here, is a promising experimental approach to

19 investigate these questions. Our investigations of the sandcastle worm undersea adhesive is

20 motivated, in part, by its potential to guide the design of useful and practical underwater setting

21 adhesives, paints, and membranes.[33,36,37,43,44] Work is in progress to incorporate additional

22 features of the entire sandcastle worm adhesive system into sophisticated biomaterials formed in

23 water. 


\section{Acknowledgements}

3 Funding from the National Science Foundation, the Office of Naval Research, and the National

4 Institutes of Health is gratefully acknowledged.

\section{References}

[1] Kirtley DW, Tanner WF. Sabellariid worms: builders of a major reef type. Journal of Sedimentary Research 1968;38:73-8.

[2] Noernberg MA, Fournier J, Dubois S, Populus J. Using airborne laser altimetry to estimate Sabellaria alveolata (Polychaeta: Sabellariidae) reefs volume in tidal flat environments. Estuarine, Coastal and Shelf Science 2010;90:93-102. doi:10.1016/j.ecss.2010.07.014.

[3] Le Cam J-B, Fournier J, Etienne S, Couden J. The strength of biogenic sand reefs: Visco-elastic behaviour of cement secreted by the tube building polychaete Sabellaria alveolata, Linnaeus, 1767. Estuarine, Coastal and Shelf Science 2011;91:333-9. doi:10.1016/j.ecss.2010.10.036.

[4] Dubois S, Reti re C, Olivier FDR. Biodiversity associated with Sabellaria alveolata (Polychaeta: Sabellariidae) reefs: effects of human disturbances. J Mar Biol Ass 2002;82:817-26. doi:10.1017/S0025315402006185.

[5] Dubois S, Barillé L, Cognie B, Beninger PG. Particle capture and processing mechanisms in Sabellaria alveolata·(Polychaeta: Sabellariidae). Marine Ecology Progress Series 2005;301:159-71.

[6] Jensen RA, Morse DE. Chemically induced metamorphosis of polychaete larvae in both the laboratory and ocean environment. Journal of Chemical Ecology 1990;16:911-30.

[7] Stewart R, Weaver J, Morse D, Waite J. The tube cement of Phragmatopoma californica: a solid foam. J Exp Biol 2004;207:4727-34.

[8] Oparin AI. The origin of life. London: The Macmillan Company; 1938.

[9] Hyman AA, Simons K. Phase Transitions in Cells-- Beyond Oil and Water. Science 2012;337:1047-9. doi:10.1126/science.1227528.

[10] Sear RP. Phase separation of equilibrium polymers of proteins in living cells. Faraday Discuss 2008;139:21-34. doi:10.1039/b713076g. 
[11] Sear RP. Dishevelled: a protein that functions in living cells by phase separating. Soft Matter 2007;3:680-5. doi:10.1039/b618126k.

[12] Brangwynne CP, Eckmann CR, Courson DS, Rybarska A, Hoege C, Gharakhani J, et al. Germline P granules are liquid droplets that localize by controlled dissolution/condensation. Science 2009;324:1729-32. doi:10.1126/science.1172046.

[13] Brangwynne CP, Mitchison TJ, Hyman AA. Active liquid-like behavior of nucleoli determines their size and shape in Xenopus laevis oocytes. Proc Natl Acad Sci 2011;108:4334-9. doi:10.1073/pnas.1017150108.

[14] Vovelle J. Le Tube de Sabellaria alveolata (L.) -- Annélide polychète Hermellidae -- et son ciment. 1965.

[15] Wang CS, Stewart RJ. Localization of the bioadhesive precursors of the sandcastle worm, Phragmatopoma californica (Fewkes). J Exp Biol 2012;215:351-61. doi:10.1242/jeb.065011.

[16] Becker PT, Lambert A, Lejeune A, Lanterbecq D, Flammang P. Identification, Characterization, and Expression Levels of Putative Adhesive Proteins From the TubeDwelling Polychaete Sabellaria alveolata. The Biological Bulletin 2012;223:217-25.

[17] Stevens MJ, Steren RE, Hlady V, Stewart RJ. Multiscale structure of the underwater adhesive of Phragmatopoma californica: a nanostructured latex with a steep microporosity gradient. Langmuir 2007;23:5045-9. doi:10.1021/la063765e.

[18] Wang C-S, Stewart RJ. Multipart Copolyelectrolyte Adhesive of the Sandcastle Worm, Phragmatopoma californica(Fewkes): Catechol Oxidase Catalyzed Curing through Peptidyl-DOPA. Biomacromolecules 2013;14:1607-17. doi:10.1021/bm400251k.

[19] Endrizzi B, Stewart R. Glueomics, An Expression Survey of the Adhesive Gland of the Sandcastle Worm. The Journal of Adhesion 2009;85:546-59.

[20] Waite J, Jensen R, Morse D. Cement precursor proteins of the reef-building polychaete Phragmatopoma californica (Fewkes). Biochemistry 1992;31:5733-8.

[21] Wang CS, Stewart RJ. Multipart copolyelectrolyte adhesive of the sandcastle worm, Phragmatopoma californica (Fewkes): catechol oxidase catalyzed curing through peptidyl-DOPA. Biomacromolecules 2013. doi:10.1021/bm400251k.

[22] Zhao H, Sun C, Stewart RJ, Waite JH. Cement proteins of the tube-building polychaete Phragmatopoma californica. J Biol Chem 2005;280:42938-44. 
doi:10.1074/jbc.M508457200.

[23] Wang CS, Svendsen KK, Stewart RJ. Morphology of the Adhesive System in the Sandcastle Worm, Phragmatopoma californica. In: Byern von J, Grunwald I, editors. Biological Adhesive Systems, Vienna: Springer Science \& Business Media; 2011, p. 305. doi:10.1007/978-3-7091-0286-2.

[24] Simple adhesive coacervates and methods of making and using thereof. US9173971, n.d.

[25] Rowlinson JS, Widom B. Molecular Theory of Capillarity. Courier Corporation; 2013.

[26] Butt H-J, Kappl M. Normal capillary forces. Adv Colloid Interface Sci 2009;146:48-60. doi:10.1016/j.cis.2008.10.002.

[27] Stevens TH, Forgac M. Structure, function and regulation of the vacuolar $(\mathrm{H}+)$-ATPase. Annual Review of Cell and Developmental Biology 1997;13:779-808. doi:10.1146/annurev.cellbio.13.1.779.

[28] Alberts B, Bray D, Lewis J. Molecular Biology of the Cell (3rd edn). Trends in ...; 1995.

[29] Koenhen DM, Mulder MHV, Smolders CA. Phase separation phenomena during the formation of asymmetric membranes. J Appl Polym Sci 1977;21:199-215.

[30] Xu M, Lewis JA. Phase behavior and rheological properties of polyamine-rich complexes for direct-write assembly. Langmuir 2007;23:12752-9. doi:10.1021/1a702249u.

[31] Hess ST, Girirajan TPK, Mason MD. Ultra-High Resolution Imaging by Fluorescence Photoactivation Localization Microscopy. Biophysical Journal 2006;91:4258-72. doi:10.1529/biophysj.106.091116.

[32] Stewart RJ, Ransom TC, Hlady V. Natural underwater adhesives. J Polym Sci B Polym Phys 2011;49:757-71. doi:10.1002/polb.22256.

[33] Stewart RJ, Wang CS, Shao H. Complex coacervates as a foundation for synthetic underwater adhesives. Adv Colloid Interface Sci 2011;167:85-93. doi:10.1016/j.cis.2010.10.009.

[34] Vincent J. Structural Biomaterials. Princeton University Press; 2012.

[35] Mow VC, Guo XE. Mechano-Electrochemical Properties Of Articular Cartilage: Their Inhomogeneities and Anisotropies. Annu Rev Biomed Eng 2002;4:175-209. doi:10.1146/annurev.bioeng.4.110701.120309.

[36] Shao H, Stewart R. Biomimetic underwater adhesives with environmentally triggered 
setting mechanisms. Adv Mater 2010;22:729-33.

[37] Jones JP, Sima M, O'Hara RG, Stewart RJ. Water-Borne Endovascular Embolics Inspired by the Undersea Adhesive of Marine Sandcastle Worms. Advanced Healthcare Materials 2016. doi:10.1002/adhm.201500825.

[38] Lubarsky M, Ray CE, Funaki B. Embolization agents-which one should be used when? Part 1: large-vessel embolization. Semin Intervent Radiol 2009;26:352-7. doi:10.1055/s0029-1242206.

[39] Lubarsky M, Ray C, Funaki B. Embolization agents-which one should be used when? Part 2: small-vessel embolization. Semin Intervent Radiol 2010;27:99-104. doi:10.1055/s-0030-1247891.

[40] Solomon EI, Sundaram UM, Machonkin TE. Multicopper Oxidases and Oxygenases. Chemical Reviews 1996;96:2563-606.

[41] Alexander JE, Corcoran EF. The distribution of copper in tropical seawater. Limnology and Oceanography 1967:236-42.

[42] Kenten RH. Latent phenolase in extracts of broad-bean (Vicia fabaL.) leaves. 2. Activation by anionic wetting agents. Biochem J 1958;68:244-51.

[43] Shao H, Bachus KN, Stewart RJ. A water-borne adhesive modeled after the sandcastle glue of P. californica. Macromol Biosci 2009;9:464-71. doi:10.1002/mabi.200800252.

[44] Kaur S, Weerasekare GM, Stewart RJ. Multiphase adhesive coacervates inspired by the Sandcastle worm. ACS Appl Mater Interfaces 2011;3:941-4. doi:10.1021/am200082v. 